\section{Military Technical College Kobry El-Kobbah, Cairo, Egypt}

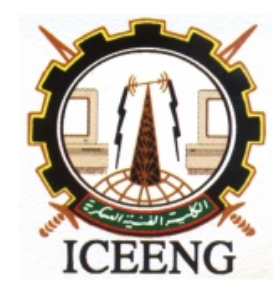

\section{$7^{\text {th }}$ International Conference on Electrical Engineering ICEENG 2010}

\title{
Real time IMU Simulator for Integrated Navigation Systems
}

\author{
By \\ $\begin{array}{llll}\text { M. Abozead * } & \text { Y. Z. Elhalwagy* } & \text { G. A. Elshikh** } & \text { I. A. Abd-El-Dayem* }\end{array}$
}

\section{Abstract:}

Nowadays, the integration of INS with GPS leads to many applications, mainly driven by the development of low-cost MEMS sensors. Then, it is not surprising that great interest has been generated toward low cost inertial sensors in the last decade. Although the basic principles of INS are quite simple, the analysis of its performance and the impact of sensor quality on the overall result are quite complex. In fact, one particular aspect of INS design is the complexity to evaluate new algorithms and innovative digital signal processing with various sensors and to access their performances in function of several kinds of mobile trajectories. Therefore, as most engineering applications, the simulation can be considered an important step to implement INS/GPS integration algorithms. Therefore, this paper exploits a simulation platform for INS/GPS integrated systems. It consists of a trajectory generator, GPS simulator; IMU simulator, and optimal estimation engine. Kalman filter algorithm plays a major role in the proposed platform. It is utilized in loosely coupled architecture as the core of optimal estimation engine, inertial sensor error models, and INS mechanizations. It also provides the facility to give a real time test. The proposed platform can be regarded as a cost-effect tool to study the techniques concerning INS/GPS integrated system. It can be used for research as well as education purposes. Several practical issues concerning the implementation of INS/GPS integration algorithms are discussed to demonstrate the capability of proposed platform.

\section{Keywords:}

Real Time Simulators, Inertial Navigation Systems, GPS/INS integration

\footnotetext{
* Egyptian Armed Forces

** SVA, Cairo, Egypt
} 


\section{Introduction:}

The current market of integrated positioning and navigation systems is clearly dominated by the Global Positioning System (GPS). The need for alternative positioning systems only arises as GPS does not work in all environments. One promising development is the emergence of Micro-Electro-Mechanical Systems (MEMS) technology. Since inertial navigation systems (INS) are capable of working in all environments where GPS has difficulties, MEMS inertial systems are used as a complement to GPS. MEMS-based INS systems provide accurate navigation data over short time intervals but suffer from accuracy degradation with time due to the combined effects of errors like noises, biases, drifts and scale factor instabilities [1]. These errors can be corrected using frequent updates from external sources like GPS derived position and velocity which forms the basis of integrated INS/GPS systems. Inertial sensor errors can be divided into two parts; random and deterministic (systematic). In order to integrate MEMS inertial sensors with GPS and to provide a continuous and reliable navigation solution, the characteristics of different error sources and the understanding of the stochastic variation of these errors are of significant importance. The random errors include bias-drifts and/or scale factor drifts that correspond to the rate at which the error in inertial sensor accumulates with time [2]. These random errors have to be modeled stochastically. The deterministic error sources include the bias and the scale factor errors which can be removed by specific calibration procedures in a laboratory environment. However, for low cost MEMS sensors, these errors are quite large and their repeatability is typically poor because of the environmental dependence, especially temperature, which makes frequent calibration a necessity. More explicitly, the actual value of the bias and the scale factor varies from that obtained through calibration process due to the difference between the operational and calibration temperatures [3]. Hence there is a need for development of accurate, reliable, and efficient thermal models to be used for online and post processing applications. Since these errors get accumulated with time, the position accuracy degrades if these thermal variations for both accelerometer and gyroscope biases and scale factors are not modeled and compensated [4]. In this paper, a real time simulation for testing the navigation algorithm that considered one of the main parts in the INS system is introduced. The main idea and principle of operation for the proposed simulator is presented. In section 2, an explanation for the mechanization equation that is considered the main core for the navigation states calculation are highlighted. In section 3, the main structure of the real time simulator, hardware and software requirements, system integration and interfacing, simulator performance test and analysis and the IMU errors simulation. Finally, paper terminates with withdrawn conclusion and continuation work for further developments.

\section{INS Mechanization Equation:}

The mechanization equations convert the output of the IMU, which includes rotation rates and specific force measurements, into position, velocity and attitude information. Once the angular rates and accelerations are obtained, the calculation algorithm can be executed in the following procedure; firstly, having the initial navigation information with respect to the navigation frame, the angular rates are integrated to obtain the new orientation of the IMU. Secondly, using this information, the accelerations are rotated into the $n$-frame, where they are twice integrated to obtain velocity and position increments. We always prefer modeling body motion in the local level frame due to the following reasons. The Local-Level Frame formulation has the advantage that its axes are aligned to the local east, north and up directions. Therefore, the attitude angles (pitch, roll and azimuth) can be obtained directly as an output of the mechanization equations. Because the definition of the LocalLevel Frame is based on the normal to the reference ellipsoid, we can directly obtain geodetic coordinate differences $\{\Delta \varphi, \Delta \lambda, \Delta \mathrm{h}\}$ as output of the system. Due to Schuller's effect, the 
computational errors in the navigation parameters on the horizontal plane (North-East plane) are bound. This effect stipulates that the inertial system errors of the horizontal plane components are coupled together producing what is called a Schuller loop. Consequently, these errors oscillate with frequency called the Schuller frequency $(1 / 5000 \mathrm{~Hz})$.

\subsection{The position mechanization equation}

In terms of the time rate of change of the position components which is related to the velocity components as follows:

$$
\&=\left(\begin{array}{c}
\& \\
\& \\
h \&
\end{array}\right)=\left(\begin{array}{ccc}
1 /(M+h) & 0 & 0 \\
0 & 1 /((N+h) \cos (\varphi)) & 0 \\
0 & 0 & -1
\end{array}\right)\left(\begin{array}{l}
v_{N} \\
v_{E} \\
v_{D}
\end{array}\right)=D^{-1} v^{n}
$$

Where: $D^{-1}=\left(\begin{array}{ccc}1 /(M+h) & 0 & 0 \\ 0 & 1 /((N+h) \cos (\varphi)) & 0 \\ 0 & 0 & -1\end{array}\right)$

\subsection{The velocity mechanization equation}

In terms of time rate of change of the velocity components of a moving body in LLF can be expressed as follows:

$$
I^{\&}=R_{b}^{\lambda} f^{b}-\left(2 \Omega_{i e}^{\lambda}+\Omega_{e \lambda}^{\lambda}\right) V^{\lambda}+g^{\lambda}
$$

Where $\Omega_{i e}^{\lambda}$ and $\Omega_{e \lambda}^{\lambda}$ are the skew-symmetric matrices corresponding to $\omega_{i e}^{\lambda}$ and $\omega_{e \lambda}^{\lambda}$ respectively and they are expressed as follows:

$$
\begin{aligned}
\Omega_{i e}^{\lambda} & =\left(\begin{array}{ccc}
0 & -\omega^{e} \sin \varphi & \omega^{e} \cos \varphi \\
\omega^{e} \sin \varphi & 0 & 0 \\
-\omega^{e} \cos \varphi & 0 & 0
\end{array}\right) \\
\Omega_{e \lambda}^{\lambda} & =\left(\begin{array}{ccc}
0 & \frac{-V^{e} \tan \varphi}{N+h} & \frac{V^{e}}{N+h} \\
\frac{V^{e} \tan \varphi}{N+h} & 0 & \frac{V^{n}}{M+h} \\
\frac{-V^{e}}{N+h} & \frac{-V^{n}}{M+h} & 0
\end{array}\right)
\end{aligned}
$$




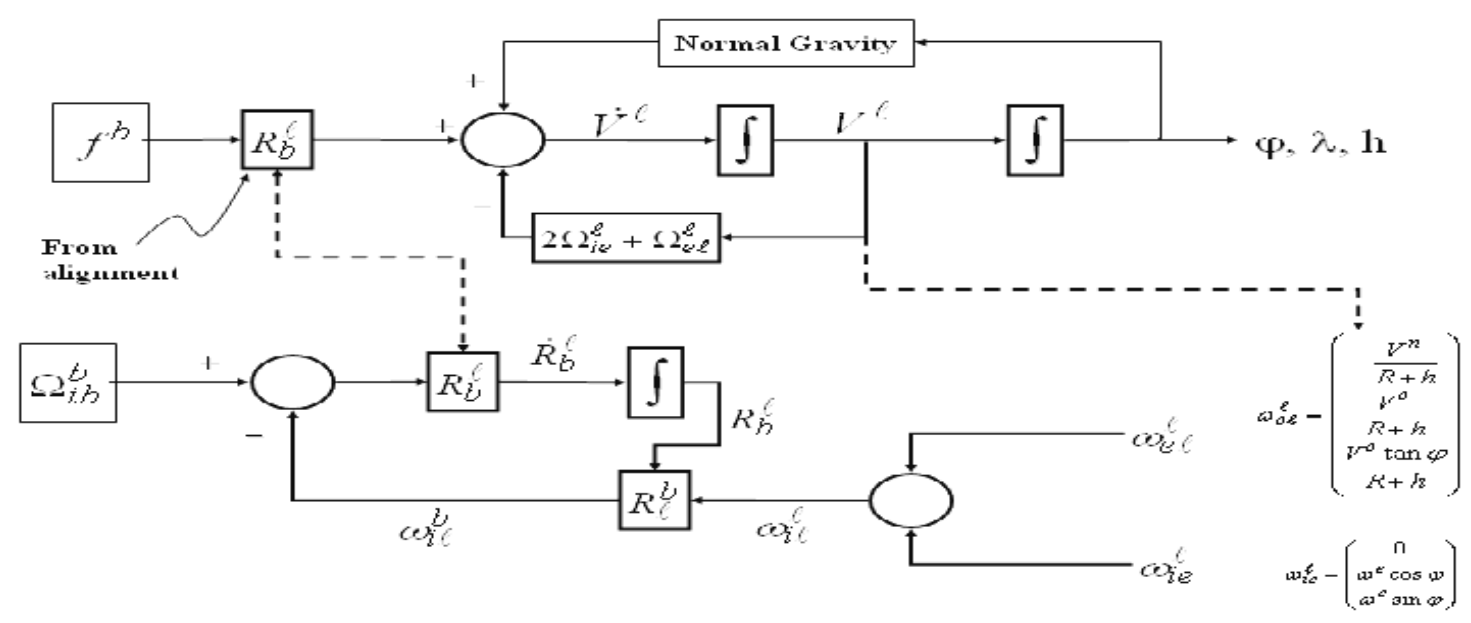

Figure (1): Over All Mechanization Equation

\section{IMU Real Time Simulator}

Simulator provides the following features; software development and validation of the designed navigation algorithm, the navigation solution in different coordinate systems as desired for the application under test, and simulating inertial sensors errors and their effect on the navigation states.

Software and hardware requirements necessary for carrying out the proposed simulator are summarized. The following paragraph denoted to illustrate these requirements.

First hardware requirements consists of two personal computers (PC), used as the host platforms for the simulator programs. Second hardware consists of the interfacing between the two computers. Interfacing between IMU data generator (PC1) and (PC2) may be realized in several types, such as standard RS-232C serial interface, RS-422 serial interface, Ethernet LAN interface or MIL-STD$1553 \mathrm{~A} / \mathrm{B}$ interface, according to the available application environment. In the IMU simulator design, software is provided to support the RS-232C type of standard interfaces. The data transferred from the flight simulator include vectors characterize the moving vehicle (attitude, position, velocity, and acceleration). These trajectory state variables can be expressed in different coordinate systems, and can be transformed into the desired navigation coordinates. Using the flight state variables, the computer code can produce complete inertial measurements for the IMU simulation.

As shown in figures 2 and 3, PC1 is considered the main hardware and software platform for the IMU simulator. The operation of the entire simulator is under the control of the computer. Data processing, user interfacing and timing control are the major functions of the host computer. The IMU measurements and sensor errors are formed in the computer by the software. The user can operate the simulator, and modify its parameters easily. The computer can also control the IMU signal generation through mode setting, parameter modification, and timing control. Electronic signal generation and injection is one of the key features of the IMU simulator, and it is also one of the most critical design issues.

As shown in figure.3.a, trajectory generator consists of a full kinematics model. It calculates a state vector consisting of several scalar variables to fully describe the trajectory of the simulated vehicle. Typically, the flight state variables include: time, translational position, velocity and acceleration, rotation matrix, angular velocity, and angular acceleration. Because the flight trajectory or states can be expressed in different coordinate systems according to mission requirements or tester preference, a 
suite of IMU measurement models expressed in different frames have been developed in this paper. The GP-soft has been adopted for trajectory generator in which trajectories for land and flight vehicle trajectories (e.g. F-16 aircraft) are simulated. The IMU raw data is calculated from the inverse operation of navigation algorithm to compute the body frame gyroscopes and accelerometers measurements from the velocity and position data generated by the trajectory generator.

To discuss the inertial sensor errors effect upon the navigation algorithm output, sources of IMU error are investigated such as bias, scale factor, scale factor error, bias drift, accelerometer random walk, and gyroscope random walk either individually and or combined. The simulation modules are carried out under MATLAB environment. The MATLAB program modules are converted to the corresponding $\mathrm{C}$ language modules by a $\mathrm{C}$-code generator in the MATLAB software. Then, the trajectory data are saved to a text files that can be loaded by the program.

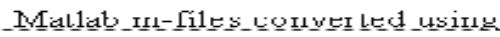

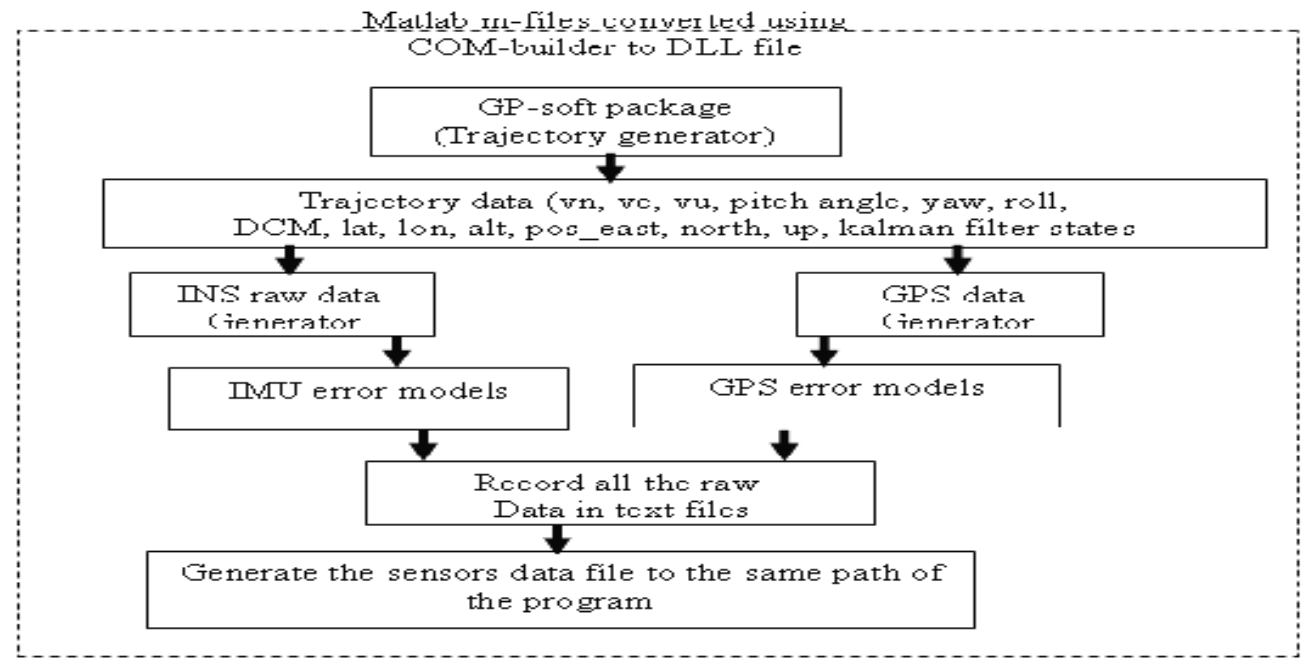

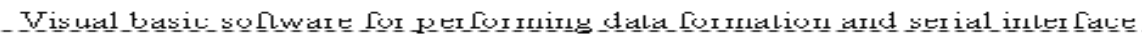

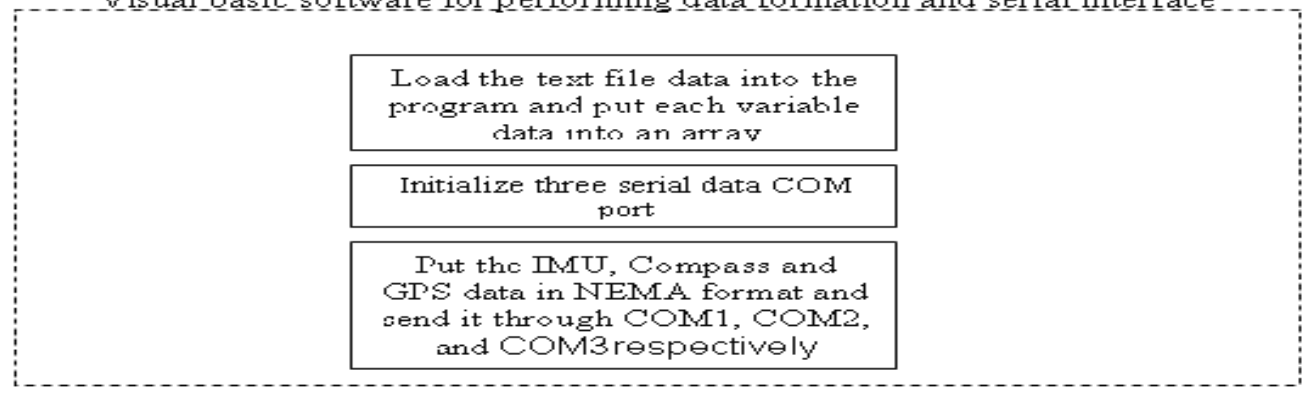

Figure (2): Real Time Simulator State Flow Diagram 


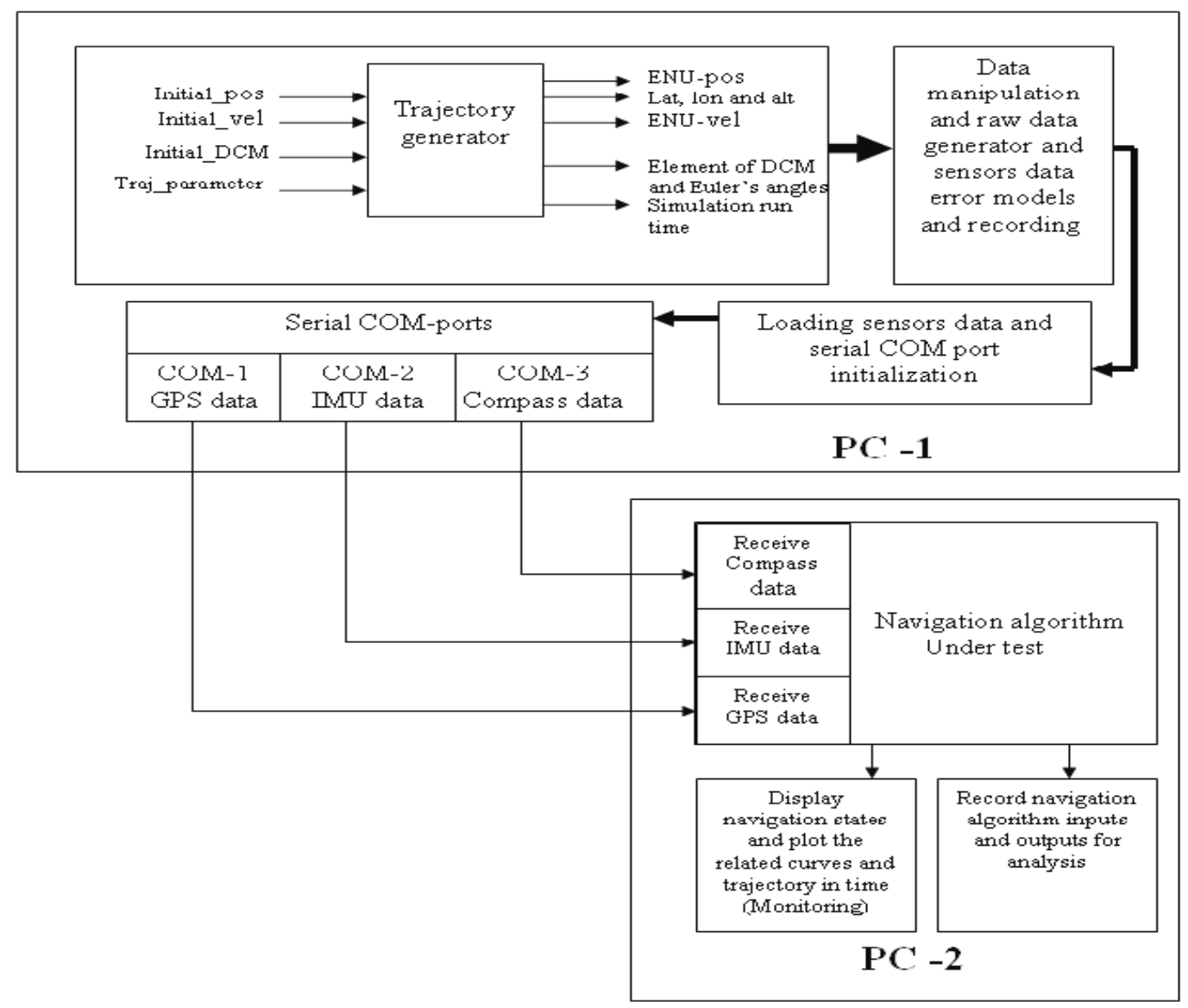

Figure (3.a): Real Time Simulator Block Diagram

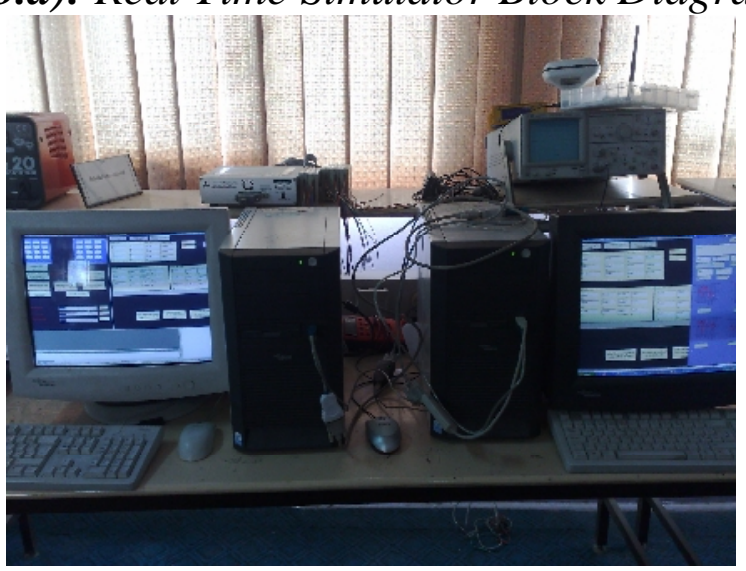

Figure (3.b): Experimental Setup for the Proposed Simulator

\section{Data formatting and interface}

The generated data text files are loaded and reformatted to NMEA format then sending the IMU message through serial COM port to match the IMU data interface hence the data can be recorded for the purpose of analysis or can be transferred to the other PC to be used as input to the navigation algorithm to be tested. The frequency and baud rate adjusted using a software program as shown in figure. $4 \mathrm{a}$. and $b$. 


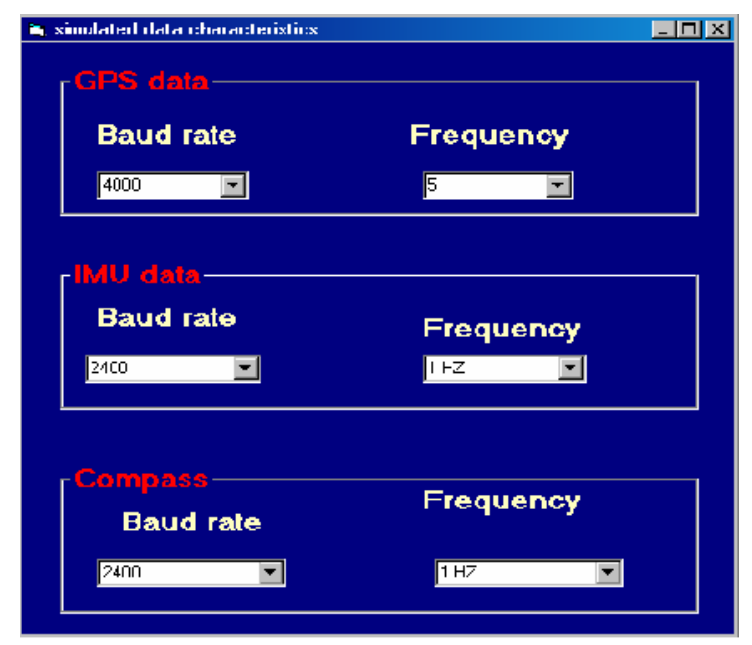

Figure (4.a): Adjusting IMU Data Transfer (Frequency and Baud Rate)

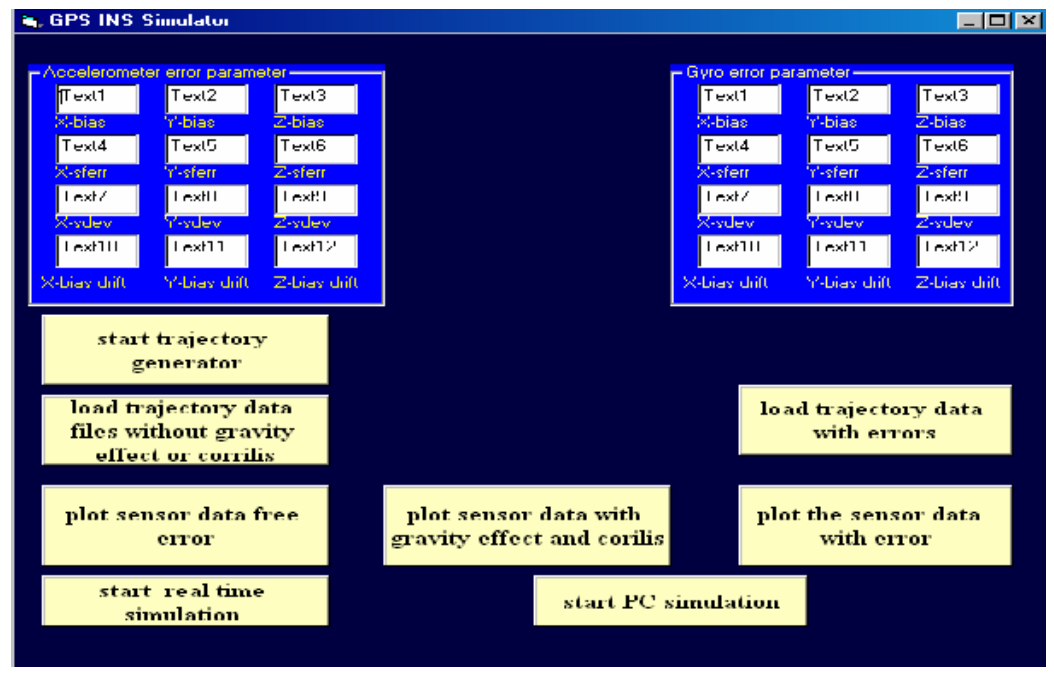

Figure (4.b): IMU Errors Setting and Simulator Main Form

\section{Inertial sensor error simulation}

The error models of the IMU are the basis for the high fidelity simulation and its closeness to reality. In the IMU simulator, the error models are devised to characterize the accuracy of the IMUs. During the simulated ground test, an error model is used to investigate the effects of the IMU sensor errors on the INS. Different types of gyroscopes and accelerometers are used in IMUs. They are based on various physical principles, and generally different types of mathematical formulations model their error characteristics. In the software design for the error models, in addition to a generic error model, we are concerned with the micro electro mechanical (MEMS) gyro and accelerometer. The IMU simulator can simulate inertial sensor errors both on an individual basis and as a combination to analyze multiple error effects. Inertial sensor errors are generally divided into two parts: deterministic and stochastic [2]. For a high-grade INS, the manufacturer typically calibrates the INS extensively and stores the compensation parameters inside the INS processor and therefore only small random errors remain. For a low performance INS, like the MEMS based INS, there are many additional error 
sources. This section uses a MEMS grade INS as an example to describe many of the sensor errors modeled in the simulator. For a MEMS inertial sensor, the deterministic part of the errors includes bias offset, scale factor (SF) error, gyro g-sensitivity, nonorthogonality, and SF non-linearity which can be roughly estimated by lab calibrations or manufacturer specifications [5]. These errors should be compensated before the INS data are used in the mechanization algorithms.

The stochastic part of MEMS inertial sensor errors includes angular random walk (ARW), velocity random walk (VRW), SF changes due to temperature and bias drift due to temperature. The random constant, the random walk and the first-order Gauss-Markov models are typically used in modeling the inertial sensor errors [6]. Table 1 presents the various types of inertial sensor errors, their characteristics, and their mathematical models [7].

Table 1 Simulated IMU errors

\begin{tabular}{|c|c|c|c|}
\hline $\begin{array}{l}\text { ERROR } \\
\text { TYPE }\end{array}$ & CHARACTERISTICS & MATHEMATICAL MODEL & $\begin{array}{l}\text { APPLIED } \\
\text { TO: }\end{array}$ \\
\hline Static bias & $\begin{array}{l}\text { These are random constant errors, i.e. } \\
\text { their initial values are random but } \\
\text { remain constant throughout the period } \\
\text { of operation of the sensors. }\end{array}$ & $\begin{array}{l}b=0 \\
\text { where } b \text { is the bias }\end{array}$ & $\begin{array}{l}\text { Accelerometer } \\
\text { and gyroscope } \\
\text { measurements }\end{array}$ \\
\hline $\begin{array}{l}\text { Scale factor } \\
\text { error }\end{array}$ & $\begin{array}{l}\text { These terms arise due to non-linearity } \\
\text { of the scale factor which is typically } \\
\text { assumed to be linear. }\end{array}$ & $\begin{array}{l}k_{n l}=k_{l}(1+x) \\
\text { where knl is the non-linear gain, } \\
\mathrm{kl} \text { is the linear gain and } \\
\mathrm{x} \text { is the non-linearity coefficient. }\end{array}$ & $\begin{array}{l}\text { Accelerometer } \\
\text { and gyroscope } \\
\text { measurements }\end{array}$ \\
\hline $\begin{array}{l}\text { Angular } \\
\text { Random } \\
\text { Walk, } \\
\text { velocity } \\
\text { random }\end{array}$ & $\begin{array}{l}\text { This error appears when random noise } \\
\text { is passed through an integrator. When } \\
\text { there is a random error }\end{array}$ & $\begin{array}{l}\omega_{R w}=a(t) / \sqrt{d t} \\
\omega_{R w} \text { is the component of } \\
\text { angular rate due to random walk } \\
\text { noise and a }(\mathrm{t}) \text { is a random } \\
\text { variable with Gaussian } \\
\text { statistical distribution. }\end{array}$ & $\begin{array}{l}\text { Gyroscope } \\
\text { measurements }\end{array}$ \\
\hline $\begin{array}{l}\text { Random } \\
\text { Noise }\end{array}$ & $\begin{array}{l}\text { This is a random signal present in the } \\
\text { output of the sensor due to different } \\
\text { reasons such as random processes in } \\
\text { front end electronic circuitry or } \\
\text { Variation in thermal characteristics. }\end{array}$ & $\begin{array}{l}x_{R}=a(t) \\
x_{R} \text { is the random noise } \\
\text { component of the output of the } \\
\text { Sensor and a (t) is a random } \\
\text { variable with a Gaussian } \\
\text { statistical distribution. }\end{array}$ & $\begin{array}{l}\text { Accelerometer } \\
\text { and gyroscope } \\
\text { measurements }\end{array}$ \\
\hline
\end{tabular}

\section{Performance test and analysis}

The correctness and efficiency of the simulator are verified at two levels; First, the individual inertial sensor errors and their combinations are verified. Second, the simulated signals are compared with real hardware INS signals collected in a field test [8].

To complete the verification of the individual errors and their combinations from the simulator, a set of vehicle trajectories and motions is designed. The designed data set involved over 200 seconds and included most of the vehicle's dynamics, such as accelerations, decelerations, static periods, turns, Uturns, tilts, and so on, as show in Figure 5. 


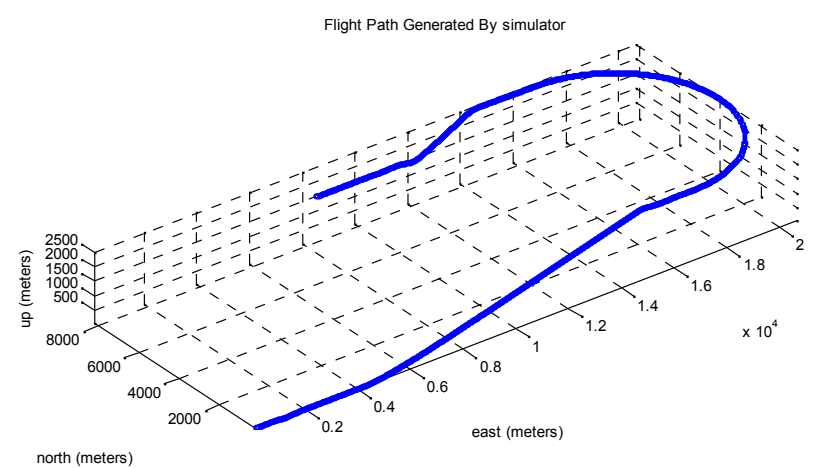

Figure (5): Simulated Trajectory

By analyzing the motions, it is obvious that the inertial sensors correctly sense the rectilinear and angular movement of the vehicle. For example, the x-axis gyro should sense the roll angle rotation. The designed roll angle changes around 100s and 150s as shown in Figure 6. In addition, the simulated velocity and the related accelerations as shown in Figure 7.
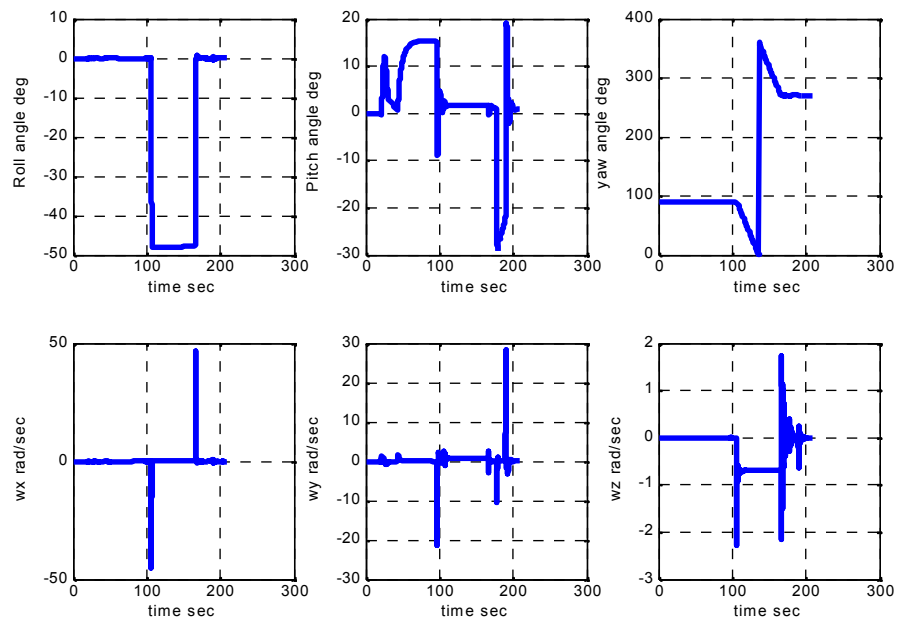

Figure (6): Simulated Attitude Angles and the Associated Angular Rates 

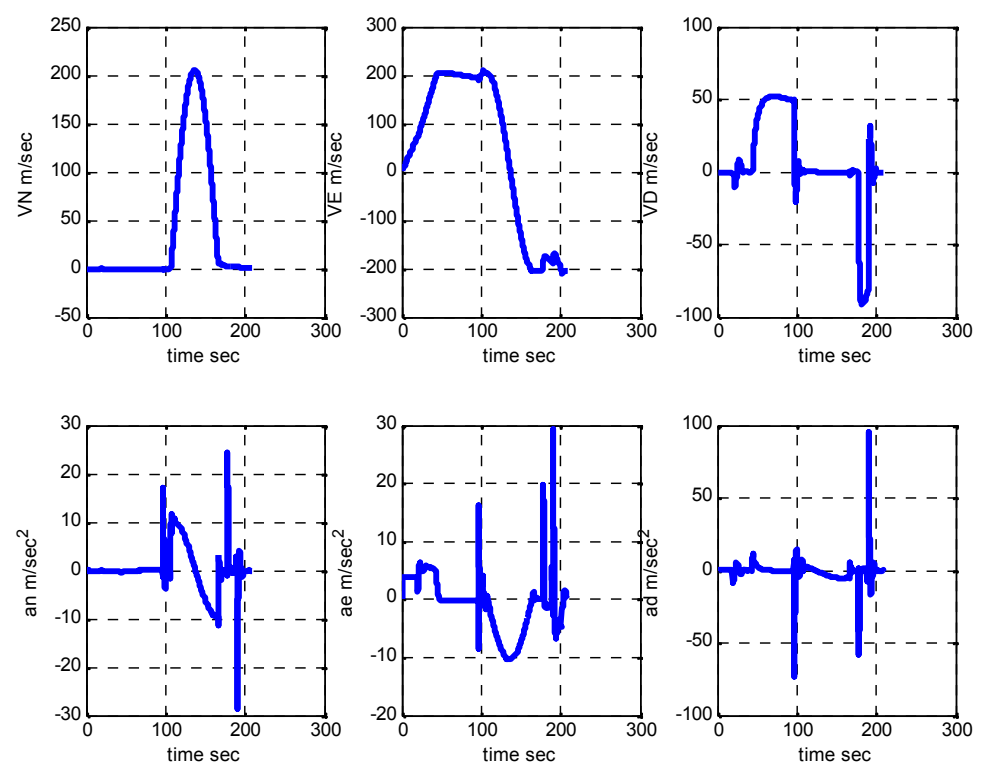

Figure (7): Simulated velocities and the related accelerations

Verification of the simulator at this raw signal level is performed based on an ADI MEMS grade INS and its parameters. The hardware ADI INS is a very low-cost MEMS INS, developed by the mobilemulti-sensor-system (MMSS) research group at the University of Calgary. The ADI INS integrates surface micromachining MEMS gyroscopes (ADXRS150) and accelerometers (ADXL105) developed by Analog Devices Inc. To compare simulated signals with those of the ADI INS, a field test was conducted around spring bank, Alberta in December 2005. Figure. 8 shows the test trajectory of the car. The trajectory was recorded by a tactical grade LN200 INS and differential GPS, which is accurate enough as the reference (true values) for the MEMS INS. The simulated INS signal of the $\mathrm{x}$-axis gyroscope is compared with that of the field test data, as shown in Figures (9-12). It is obvious that the simulated results imply adequately the real data. Small errors between simulated and real angular rate and accelerations are reported in Figures 11 and 12.

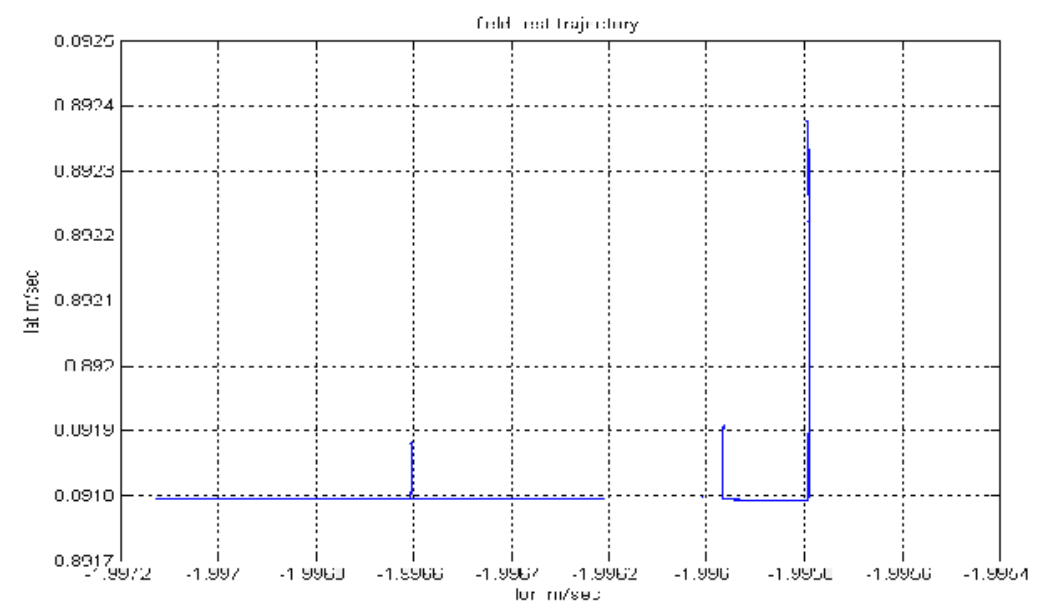

Figure (8): Field test trajectory 

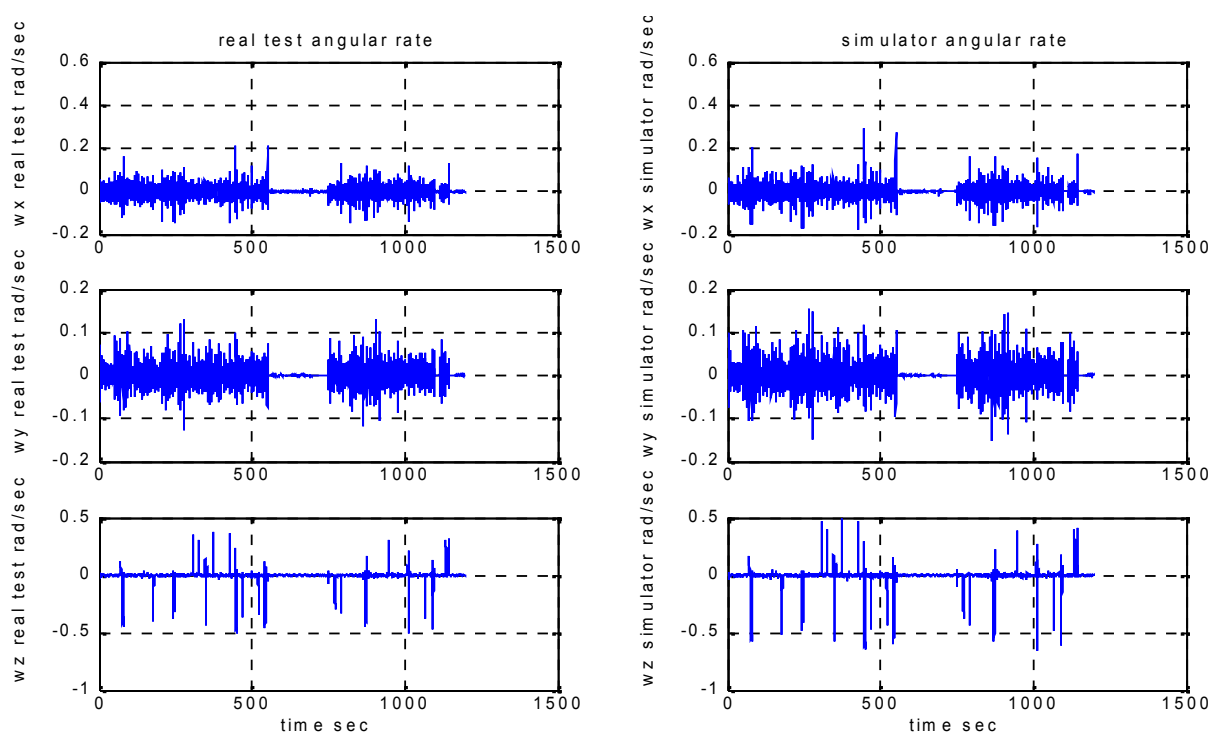

Figure (9):Real test angular rates versus simulated angular rates
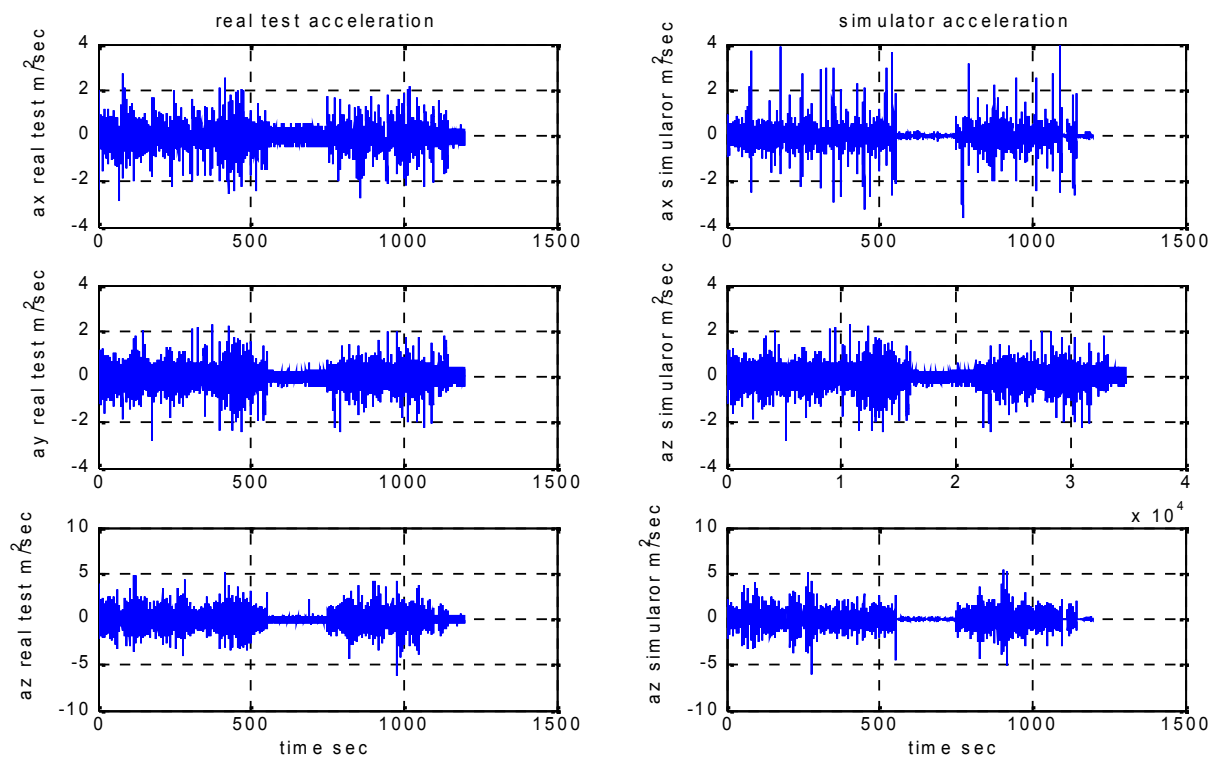

Figure (10): Real test acceleration versus simulated accelerations 

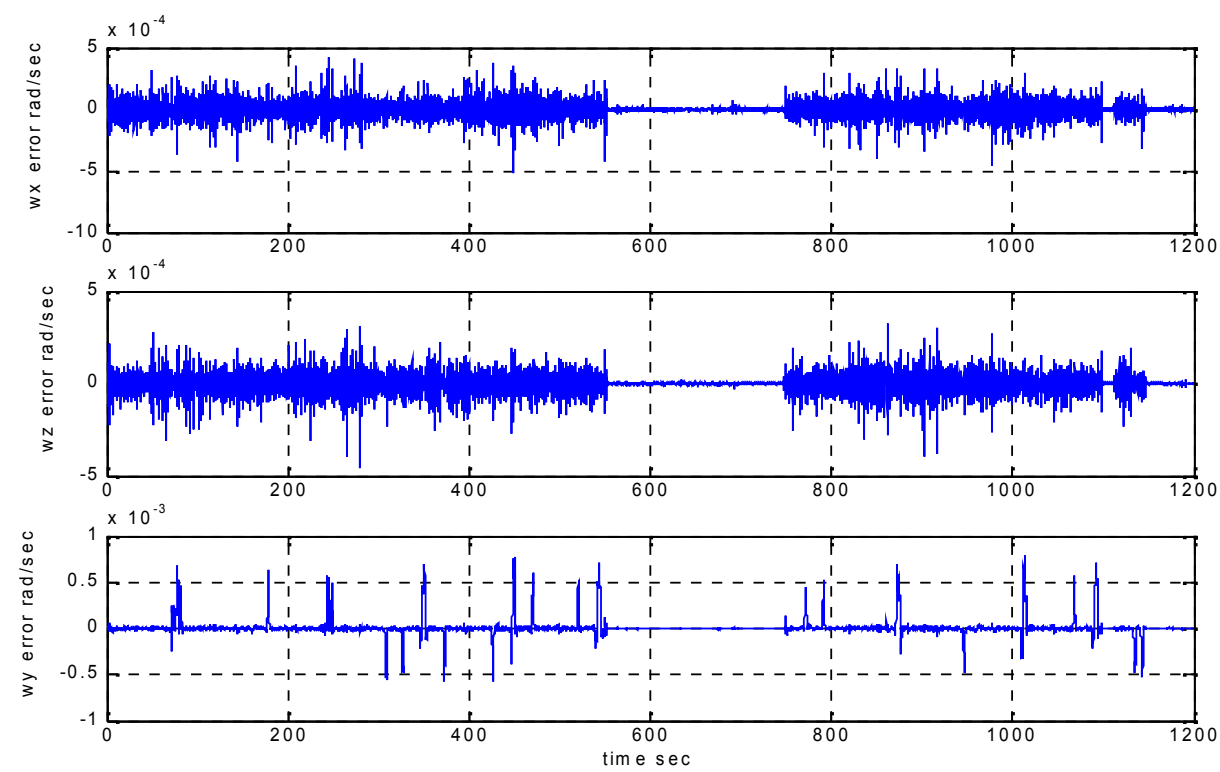

Figure (11): Error between real and simulated angular rates
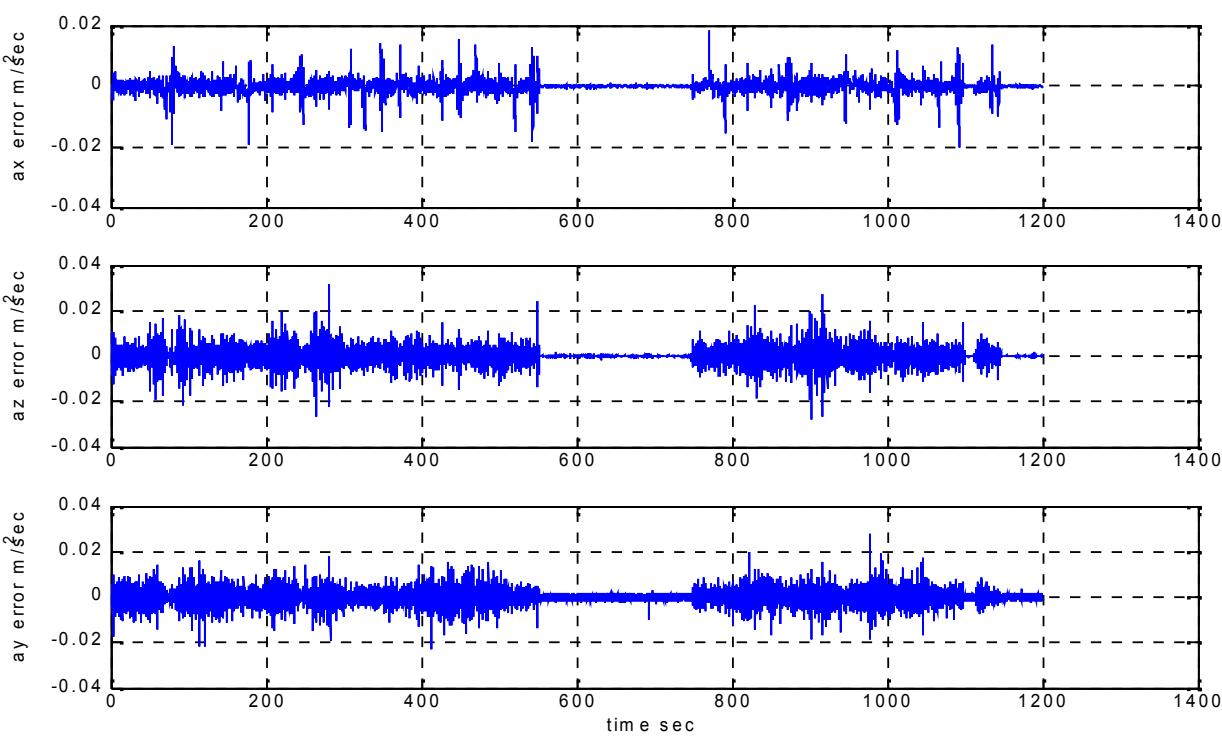

Figure (12): Errors between real and simulated accelerations

\section{Navigation Algorithm Evaluation Using Real Time Simulator}

The evaluation of the navigation algorithm established through generating several paths using the simulator trajectory generator and uses the generated IMU data as an input to the navigation algorithm then comparing the output of the designed navigation algorithm and the reference generated trajectory. The designed trajectories involved over 200 seconds and included most of the vehicle's dynamics, such as accelerations, decelerations, static periods, turning maneuvers (U-turns), tilts, and so on. The simulated trajectory IMU data is free of any inertial sensors errors for testing the performance of 
the established navigation algorithm and calculation stability under high IMU data rate up to $100 \mathrm{~Hz}$. As shown in Figures from Figure.13 to Figure.19 the comparison of the navigation algorithm output and the corresponding reference generated trajectory data are illustrated. The errors generated due to the navigation algorithm computation are shown in Figures 15, 17, 19 then the algorithm performance from calculation and stability point of view is trusted but the generated errors appears due to the Corolis effect compensation, gravity estimation model, and numerical integration errors.

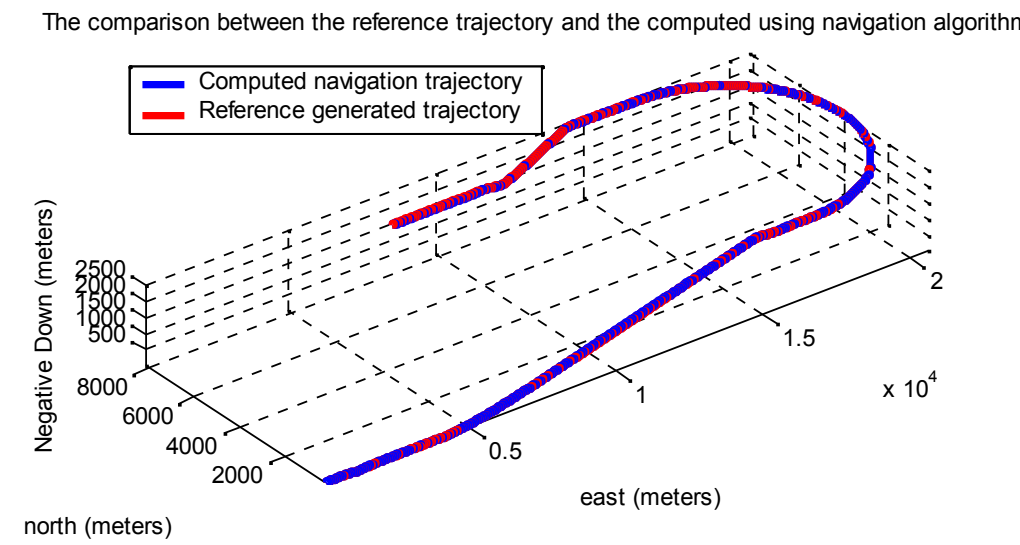

Figure (13): Simulated and computed trajectories

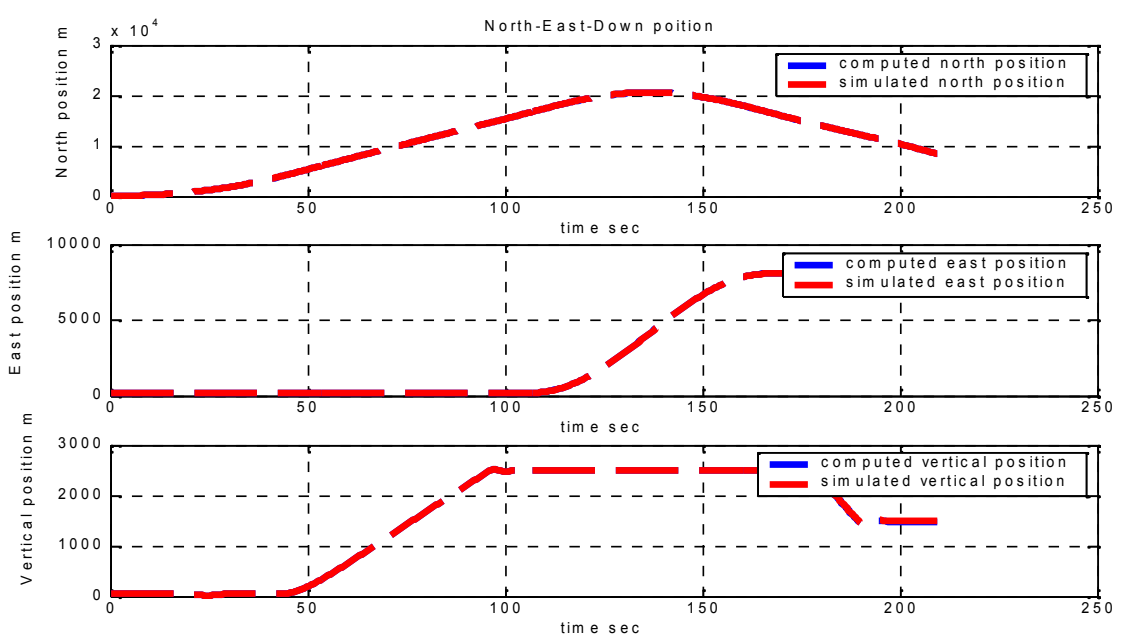

Figure (14): Simulated and computed velocity components 


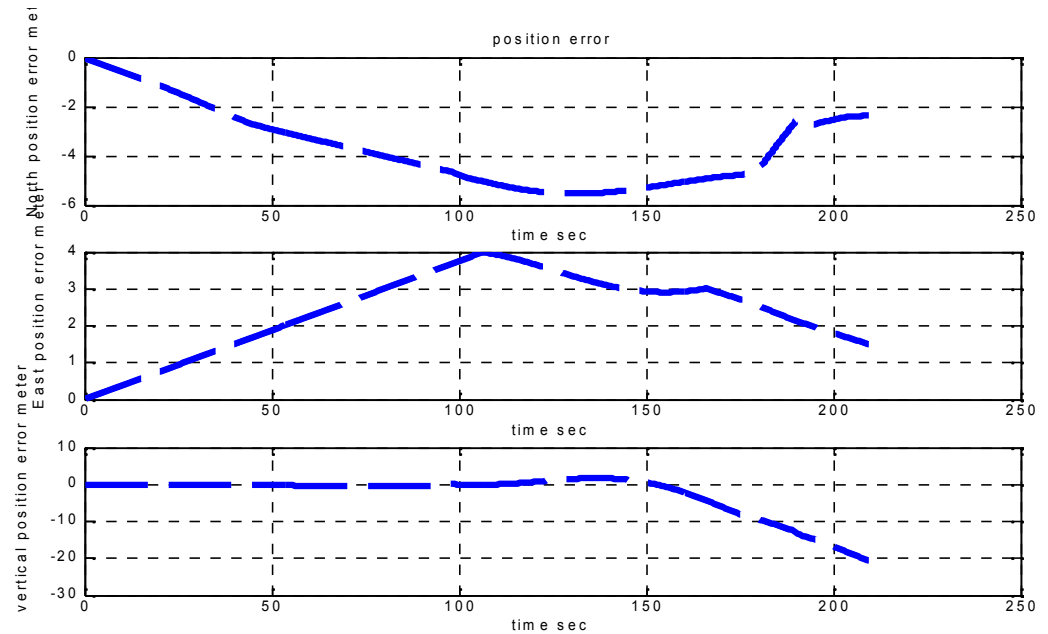

Figure (15): Error between simulated and computed position components

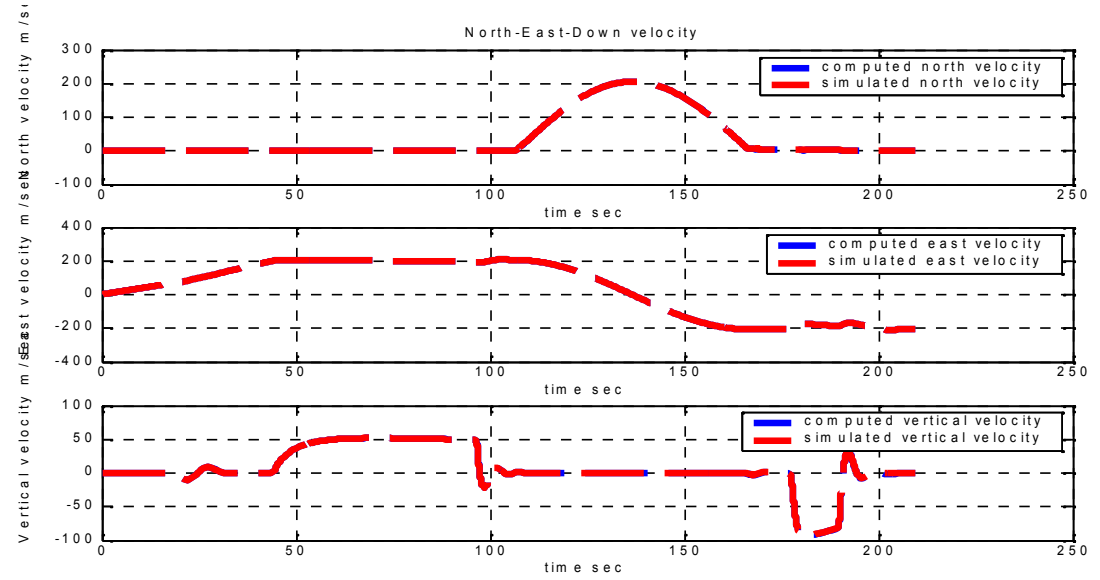

Figure (16): Simulated and computed velocity components

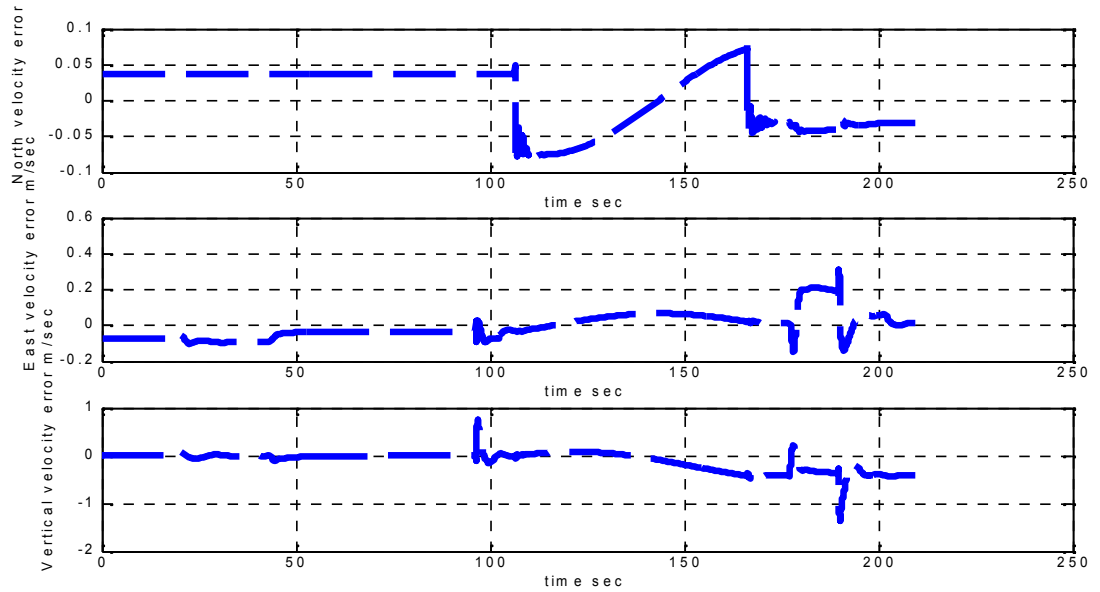

Figure (17): Errors between Simulated and computed velocity components 

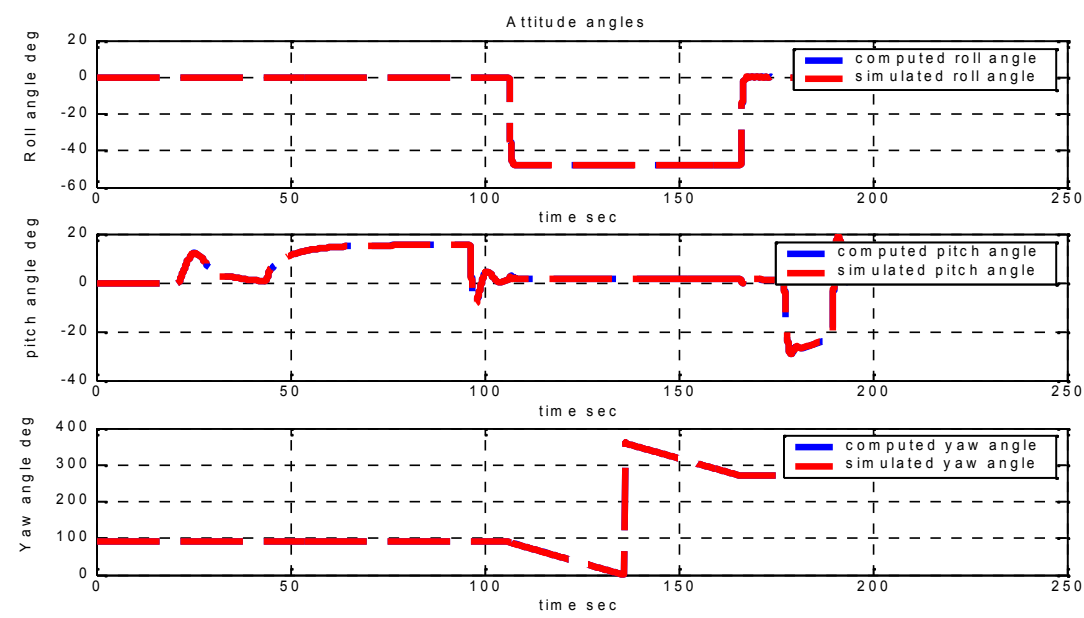

Figure (18): Simulated and computed attitude angles

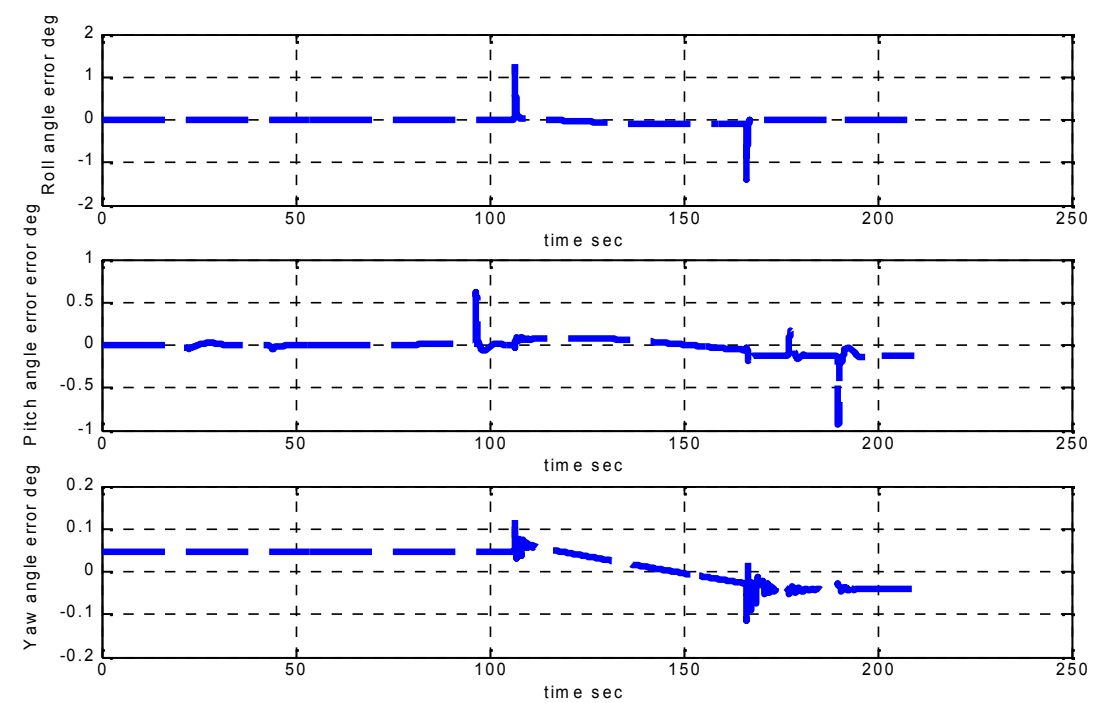

Figure (19): Error between simulated and computed attitude angles

\section{GPS simulation}

The complete simulation of GPS receiver is very difficult because of the required hardware for receiving the signals transmitted from the satellites. The GPS simulation takes the job of designing a path generator for testing the GPS navigation algorithm (navigation module). The path consists of a software programs for; 1 . Vehicle path (trajectory) generator: path consists of straight segments joined by constant-radius turns. 2 . Generate matrix of positions of satellites which are visible to the user at location user xyz. In ECEF. 3. Generate vector of 'measured' pseudoranges and accumulated delta ranges (i.e., integrated Doppler) to all visible satellites. 4. Simulating error sources affect the pseudorange measurements Thermal Noise, Tropospheric error, selective availability, Multipath error, and Ionospheric error. The simulated pseudoranges and satellite positions data transferred to the GPS navigation algorithm under test as shown in Figure20. 


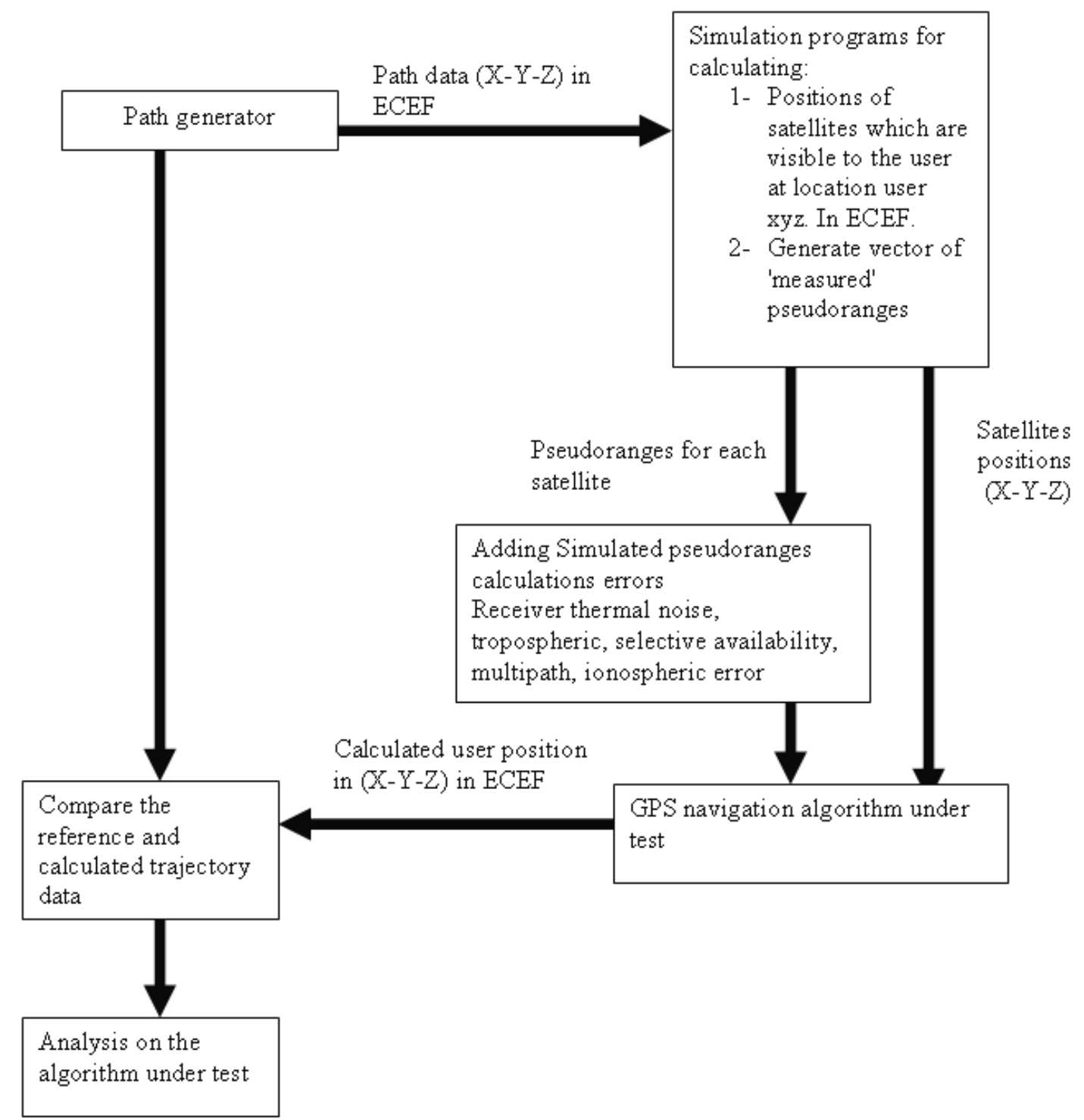

Figure (20): Simulation procedure for testing the GPS navigation algorithms

The following steps summarize user position solution in the GPS receiver:

1- Choose a nominal position and user clock bias $x u 0, y u 0, z u 0, b u 0$ to represent the initial condition. For example, the position can be the center of the earth and the clock bias zero. In other words, all initial values are set to be zero.

2- Calculate the pseudorange $\rho_{i}$. These $\rho_{i}$ values will be different from the measured values. The difference between the measured values and the calculated values is $\delta \rho_{i}$.

3- Use the calculated $\rho_{i}$ to calculate $\alpha_{i 1} \alpha_{i 2}, \alpha_{i 3}$.

4- Find $\delta x_{u}, \delta y_{u}, \delta z_{u}$, and $\delta b_{u}$.

5- From the absolute values of $\delta x_{u}, \delta y_{u}, \delta z_{u}$, and $\delta b_{u}$, calculate $\delta v$.

6- Compare $\delta v$ with an arbitrarily chosen threshold; if $\delta v$ is greater than the threshold, the 
following steps will be needed:

7- Add these values $\mathrm{d} x u, \mathrm{~d} y u, \mathrm{~d} z u, \mathrm{~d} b u$ to the initial chosen position $x u 0, y u 0, z u 0$, and the clock bias $b u 0$; a new set of positions and clock bias can be obtained and they will be expressed as $x u 1, y u 1, z u 1, b u 1$. These values will be used as the initial position and clock bias in the following calculations.

8- Repeat the procedure from 1 to 7 , until $\delta v$ is less than the threshold. The final solution can be considered as the desired user position and clock bias, which can be expressed as $x u, y u, z u, b u$. In general, the $\delta v$ calculated in the above iteration method will keep decreasing rapidly. Depending on the chosen threshold, the iteration method usually can achieve the desired goal in less than 10 iterations [9].

\section{Simulation Results}

The purpose of simulation is testing the GPS navigation algorithm and showing the effect of several errors affecting the pseudorange measurements in the GPS receiver so that it established through six runs. The first is for testing the proposed GPS navigation algorithm. The remains five runs are for showing the different GPS errors and their effect on the position calculation. Figure 22 shows the simulated trajectory for testing the GPS navigation algorithm and the output trajectory. The recorded time history of the errors as shown in Figures 23, 24 give a satisfactorily results for the utilized navigation algorithm.

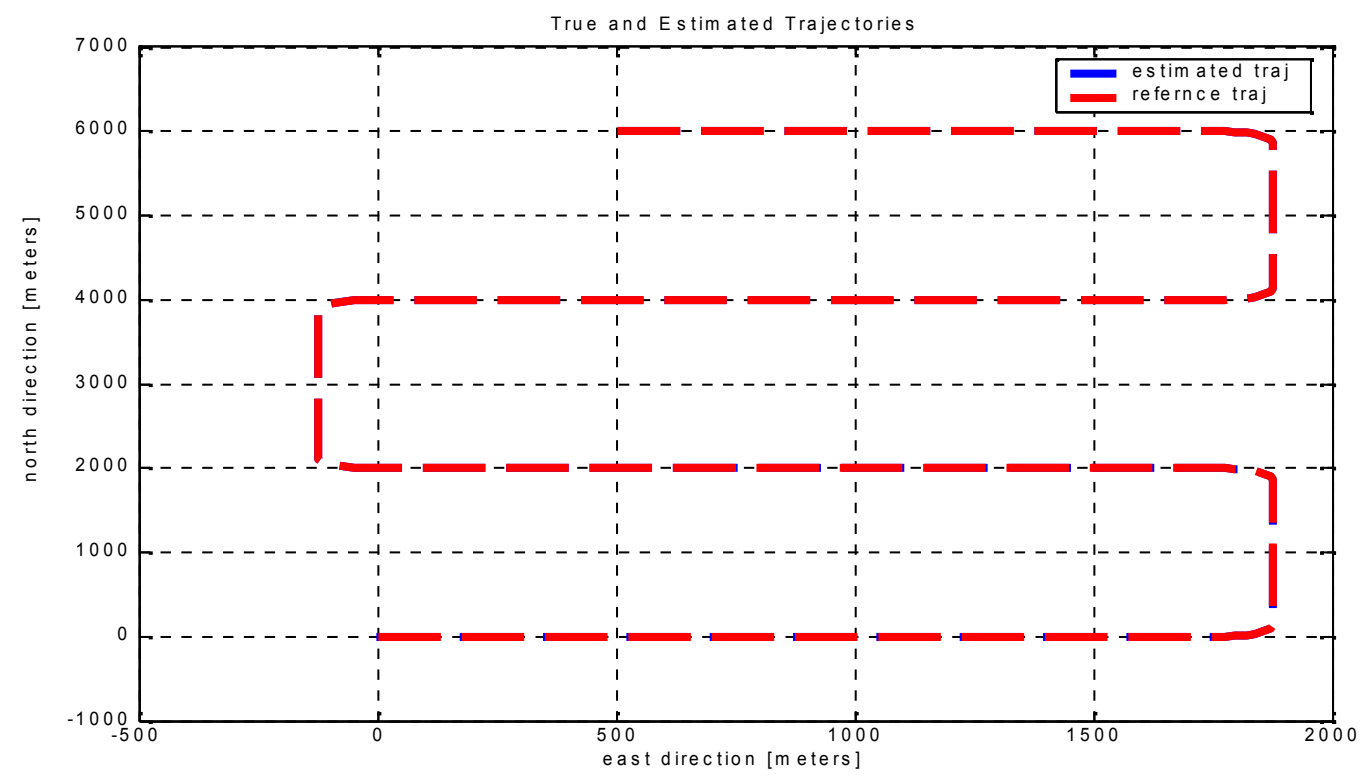

Figure (21): Estimated and reference user positions trajectories for no errors 


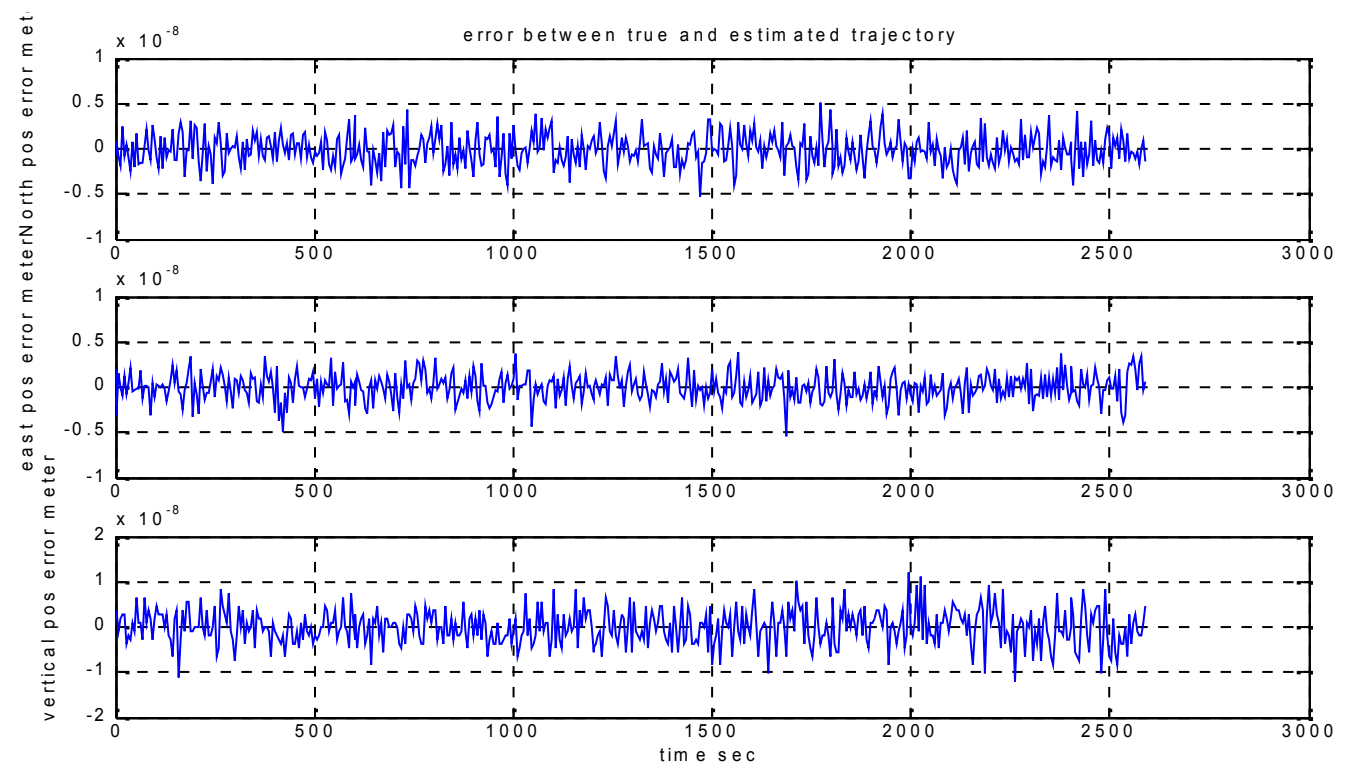

Figure (22): Errors between the simulated and reference user positions

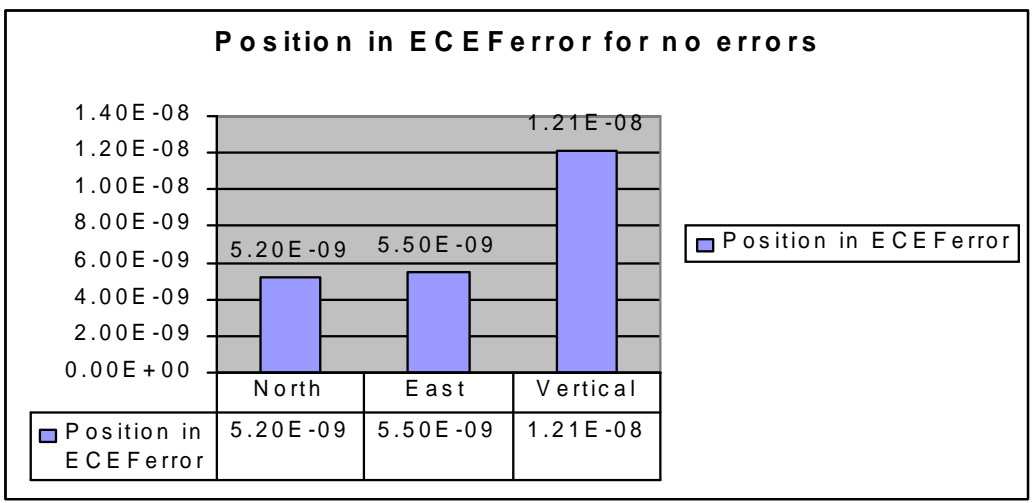

Figure (23): Errors between the estimated and reference user positions

Finally we have now a GPS system on COM-port and IMU in the other COM-port then we use the interfacing board for connecting the two cables from PC1 to PC2 and then test the previously developed aided navigation algorithm. Under field test, the simulation provide a kalman filter algorithm as an adjustable function to be used on PC-2 as it has been presented earlier in figure (3.a).

\section{Conclusions:}

In this paper, a real time simulation for testing the navigation algorithm that considered one of the main parts in the INS system is carried out. The main hardware and software requirement for building up the real time IMU simulator along with computational platform are deeply investigated and analyzed. The system integration and interfacing associated with simulator performance test and evaluation are studied where simulation and experimental results showed satisfactorily behavior of the overall system. The carried out setup is an in progress testing facility for educational and R\&D purposes. A complete simulation of IMU and partial GPS real simulators has been proposed in this paper for the task of testing various INS navigation algorithm only or integrated GPS/INS algorithms, 
and a complete simulation for the IMU errors and their effects upon navigation states and navigation parameters have been investigated.

\section{References:}

[1] Godha, S. "Performance Evaluation of Low Cost MEMS-Based IMU Integrated With GPS for Land Vehicle Navigation Application", MSc Thesis, Department of Geomatics Engineering, The University of Calgary, Canada, (2006).

[2] El-Sheimy, N. "Inertial Techniques and INS/DGPS Integration", ENGO 623 Course Notes, Department of Geomatics Engineering, University of Calgary, Canada 2006.

[3] Abdel-Hamid.W, "Accuracy Enhancement of Integrated MEMS-IMU/GPS Systems for Land Vehicular Navigation Applications", Ph.D. thesis, The University of Calgary, Alberta, January (2005).

[4] A.O. Salytcheva,“ Medium Accuracy INS/GPS Integration in Various GPS Environments," M.Sc. thesis, UCGE Report \#20200, Department of Geomatics Engineering, University of Calgary, Canada, 2004

[5] D.H. Titterton and J. L., Weston., "Strapdown inertial navigation technology", London, U.K.: Peter Peregrinus Ltd, (1997).

[6] Eun-Hwan Shin, "Estimation Techniques for Low-Cost Inertial Navigation", Ph.D. thesis, The University of Calgary, Alberta, May (2005).

[7] I.B.UMAR, "Improved integrity algorithms for integrated GPS/INS systems in the presence of slowly growing errors"PHD thesis Department of Civil and invironmental Engineering Imperial College London, London,

[8] Yong Yang, " Tightly Coupled MEMS INS/GPS Integration with INS Aided Receiver Tracking Loops", Msc. Thesis Department of Geomatics Engineering, The University of Calgary, Alberta, May 2008.

[9] JAMES BAO-YEN TSUI., "Fundamentals of Global Positioning System Receivers A Software Approach", New York: John Wiley and Sons Inc, (2000). 